\title{
SiMYB56 Confers Drought Stress Tolerance in Transgenic Rice by Regulating Lignin Biosynthesis and ABA Signaling Pathway
}

\author{
Weiya $X u^{1}$, Wensi Tang ${ }^{1}$, Chunxiao Wang ${ }^{1}$, Linhao Ge ${ }^{1}$, Jianchang Sun ${ }^{2}$, Xin Qi ${ }^{1}$, \\ Zhang He ${ }^{1}$, Yongbin Zhou ${ }^{1}$, Jun Chen ${ }^{1}$, Zhaoshi X ${ }^{1}$, You-Zhi Ma ${ }^{1 *}$ and Ming Chen ${ }^{1 *}$ \\ ${ }^{1}$ National Key Facility for Crop Genetic Resources and Genetic Improvement, Key Laboratory of Crop Genetics \\ and Breeding, Ministry of Agriculture, Institute of Crop Science, Chinese Academy of Agricultural Sciences, Beijing, China, \\ ${ }^{2}$ Institute of Crop Sciences, Ningxia Academy of Agriculture and Forestry Sciences, Yongning, China
}

OPEN ACCESS

Edited by:

Vicent Arbona

Jaume I University, Spain

Reviewed by:

Manoj Prasad,

National Institute of Plant Genome

Research (NIPGR), India

Kazuo Nakashima,

Japan International Research Center

for Agricultural Sciences (JIRCAS),

Japan

*Correspondence:

You-Zhi Ma

mayouzhi@caas.cn

Ming Chen

chenming02@caas.cn

Specialty section:

This article was submitted to

Plant Abiotic Stress,

a section of the journal

Frontiers in Plant Science

Received: 30 March 2020

Accepted: 18 May 2020

Published: 18 June 2020

Citation:

Xu W, Tang W, Wang C, Ge L, Sun J, Qi X, He Z, Zhou Y, Chen J, Xu Z, Ma Y-Z and Chen M (2020)

SiMYB56 Confers Drought Stress

Tolerance in Transgenic Rice by

Regulating Lignin Biosynthesis

and ABA Signaling Pathway.

Front. Plant Sci. 11:785.

doi: 10.3389/fp/s.2020.00785
Foxtail millet (Setaria italica) originated in China and is generally cultivated in arid and barren soil. Through long-term harsh environmental selection, foxtail millet has acquired significant drought resistance. However, the molecular mechanism of foxtail millet drought resistance is still unknown. Here, we identified a drought-induced R2R3-MYB transcription factor SiMYB56 in foxtail millet. Overexpression of SiMYB56 significantly enhances tolerance to drought stress in transgenic rice plants at both the vegetative and the reproductive stage and has no adverse effect on its normal growth. Compared with wild-type controls, SiMYB56-overexpressing rice plants had lower MDA content and higher lignin content under drought conditions. Quantitative real-time PCR and Transcriptional activity assays demonstrated that SiMYB56 could activate expression of lignin biosynthesis genes under drought conditions. Also, we found that overexpression of SiMYB56 can led to ABA accumulation in the seeds transgenic rice plants. Further experiments showed that Overexpression of SiMYB56 can upregulate the expression of ABA synthesis and response related genes under drought conditions. In conclusion, SiMYB56 may enhance the drought resistance of transgenic rice plants by regulating lignin biosynthesis and ABA signaling pathway, making SiMYB56 a candidate gene for drought resistance improvement in gramineous crops.

Keywords: foxtail millet, R2R3-MYB transcription factor, drought tolerance, lignin biosynthesis, ABA signaling pathway

\section{INTRODUCTION}

Drought is a major environmental factor that affects the natural geographical distribution of plants, limits agricultural plant productivity, and threatens food security (Lesk et al., 2016). As a consequence of drought stress, plants suffer photosynthesis inhibition, metabolic dysfunction, and cellular structure damage (Xu et al., 2010). Foxtail millet (Setaria italica), originated from China, is an important food and fodder grain crop in arid and semi-arid regions of Asia and Africa (Barton et al., 2009; Lu et al., 2009). Small diploid genome size ( $\sim 510 \mathrm{Mb})$, self-pollination, low repetitive DNA content (30\%) and short life cycle, making it an ideal and suitable model species for genetic 
and molecular studies (Doust et al., 2009; Diao et al., 2014; Muthamilarasan and Prasad, 2015). Also, foxtail millet is an elite drought-tolerant crop, water use efficiency of foxtail millet has been shown to be higher than that of maize, wheat, and sorghum (Gu et al., 1987). Because of its excellent drought tolerance and water-use efficiency, research on the mechanisms of drought tolerance of foxtail millet have great significance for crop drought-resistance molecular breeding. However, the molecular mechanism of its drought adaptation is still not clear.

Lignin biosynthesis plays an important role in plant biotic stress resistance, especially in disease resistance ( $\mathrm{Li}$ et al., 2020), and insect resistance (Wang et al., 2017). In recent years, the role of lignin in plant response to abiotic stress has been gradually revealed. Studies have found that high levels of lignification in plants can lead to increased drought tolerance, although the associated regulatory networks have not been well elucidated ( $\mathrm{Hu}$ et al., 2009; Pereira et al., 2018). It is widely accepted that lignin biosynthesis is controlled by a multi-leveled hierarchical regulatory network involving NAC and MYB transcription factors (TFs) (Zhao and Dixon, 2011; Ohtani and Demura, 2019). Some NAC TFs, including VND17 and NST1-3, were found to serve as primary switches of this network (Kubo et al., 2005; Mitsuda et al., 2007). MYB46 and MYB83, the downstream targets of NAC proteins, are secondary switches of this network (McCarthy et al., 2009). Further downstream are some other MYB genes implicated in lignin biosynthesis regulation (Ko et al., 2014). Although a battery of R2R3-MYB TFs have been identified to be involved in lignin biosynthesis in model plants like Arabidopsis, the mechanism of lignin regulation in major crops, especially Gramineae, remains unknown.

Abscisic acid (ABA), which is the central regulator of abiotic stress resistance in plants, coordinates an array of functions enabling plants to cope with different stresses (Sah et al., 2016). Abiotic stresses, especially water deficit, induce ABA accumulation, which triggers rapid biochemical and physiological responses that enhance stress adaptation (Finkelstein, 2013; Ng et al., 2014). As the largest subfamily of MYB transcription factor family, numerous evidences have shown that R2R3-MYB transcription factors play important roles in ABA signaling under drought stress (Shinozaki and Yamaguchi-Shinozaki, 2007; Baldoni et al., 2015). In Arabidopsis, AtMYB2 increased drought resistance by activating the expression of related genes induced by ABA under drought stress (Abe et al., 2003). AtMYB96 improves the drought resistance of transgenic Arabidopsis through activating cuticular wax biosynthesis in an ABA-dependent way (Seo et al., 2011). However, most identified R2R3-MYB transcription factors involved in $\mathrm{ABA}$ mediated plant drought resistance play roles in ABA signal transduction, and few of them are found play roles in ABA synthesis.

It has been pointed out that there are at least 209 MYB transcription factors in foxtail millet, and $68 \%$ of them function in response to stress (Muthamilarasan et al., 2014). However, no further research has been done on these transcription factors except for SiMYB3 which mediates the low nitrogen tolerance of foxtail millet (Ge et al., 2019). Here, we identified a drought-induced R2R3-MYB transcription factor in foxtail millet SiMYB56. Overexpression of SiMYB56 in rice significantly improved the drought resistance of transgenic rice throughout their entire growing season. By analyzing the regulation mechanism, we found that SiMYB56 improved the drought resistance of transgenic rice by regulating lignin biosynthesis and ABA signaling pathway, which enriches the study of functional genomics of drought resistance in foxtail millet and provides new strategies for improving the drought resistance of gramineous crops.

\section{MATERIALS AND METHODS}

\section{Plant Materials, Growth Conditions, and Stress Treatments}

For analysis of SiMYB56 expression under different stress treatments, foxtail millet (Yugu1) seeds were soaked in water, germinated at $28^{\circ} \mathrm{C}$ for 2 days, and then transferred to Hoagland solution for 2 weeks in a growth chamber (60\% relative humidity) under a 14 -hour-light $\left(21^{\circ} \mathrm{C}\right) / 10$-h-dark $\left(24^{\circ} \mathrm{C}\right)$ cycle. Next, 2 -week-old seedings were exposed to various abiotic stresses, including osmotic stress (10\% PEG6000), salinity stress ( $80 \mathrm{mM}$ $\mathrm{NaCl})$ and $\mathrm{ABA}$ presence $(100 \mu \mathrm{M} \mathrm{ABA})$. Leaves, stems, and roots were sampled at $0,1,3,6,12$, and $24 \mathrm{~h}$, and all samples were immediately frozen in liquid nitrogen and stored at $-80^{\circ} \mathrm{C}$ prior to analysis.

For phenotypic analysis of transgenic rice plants and wild-type controls under drought stress during vegetative stage, we carried out soil drought and hydroponic drought experiments. For the soil drought experiments, germinated seeds of wild-type controls (Ki) and transgenic rice plants (OE16/OE21/OE30) were transferred to soil. Then, rice seedlings were grown under a 14-hour-light $\left(30^{\circ} \mathrm{C}\right) / 10$-hour-dark $\left(26^{\circ} \mathrm{C}\right)$ cycle in the greenhouse. At the 5-leaf stage, watering was stopped until all wide-type controls wilted. Then, plants were re-watered for 7 days. After re-watering, survival rate statistics were collected.

For the hydroponic drought experiments, we treated wild-type controls and transgenic rice plants with 10\% PEG6000 to simulate osmotic stress caused by drought. Uniformly germinated seeds were sown into a 96-well plate that had its bottom removed. The plate was floated on water for one week and then transferred to Hoagland solution for another one week. For PEG treatment, 2-week-old seedlings were transferred to culture solution containing 10\% PEG6000 and incubated for another 2 weeks before physiological indexes were measured.

For phenotypic analysis of transgenic rice plants and wildtype controls under drought stress during reproductive stage, we carried out field-drought resistance experiments. In order to achieve a better effect from field drought, we chose to carry out the experiment in Ningxia province, which receives relatively little rain. Three independent $\mathrm{T} 3$ homozygous transgenic rice lines (OE16, OE21, and OE30), alongside wild-type controls (Ki), were transplanted to a paddy field at the Wanghong experimental station, Ningxia Academy of Agriculture and Forestry Sciences (Ningxia, China). A randomized design was employed with 

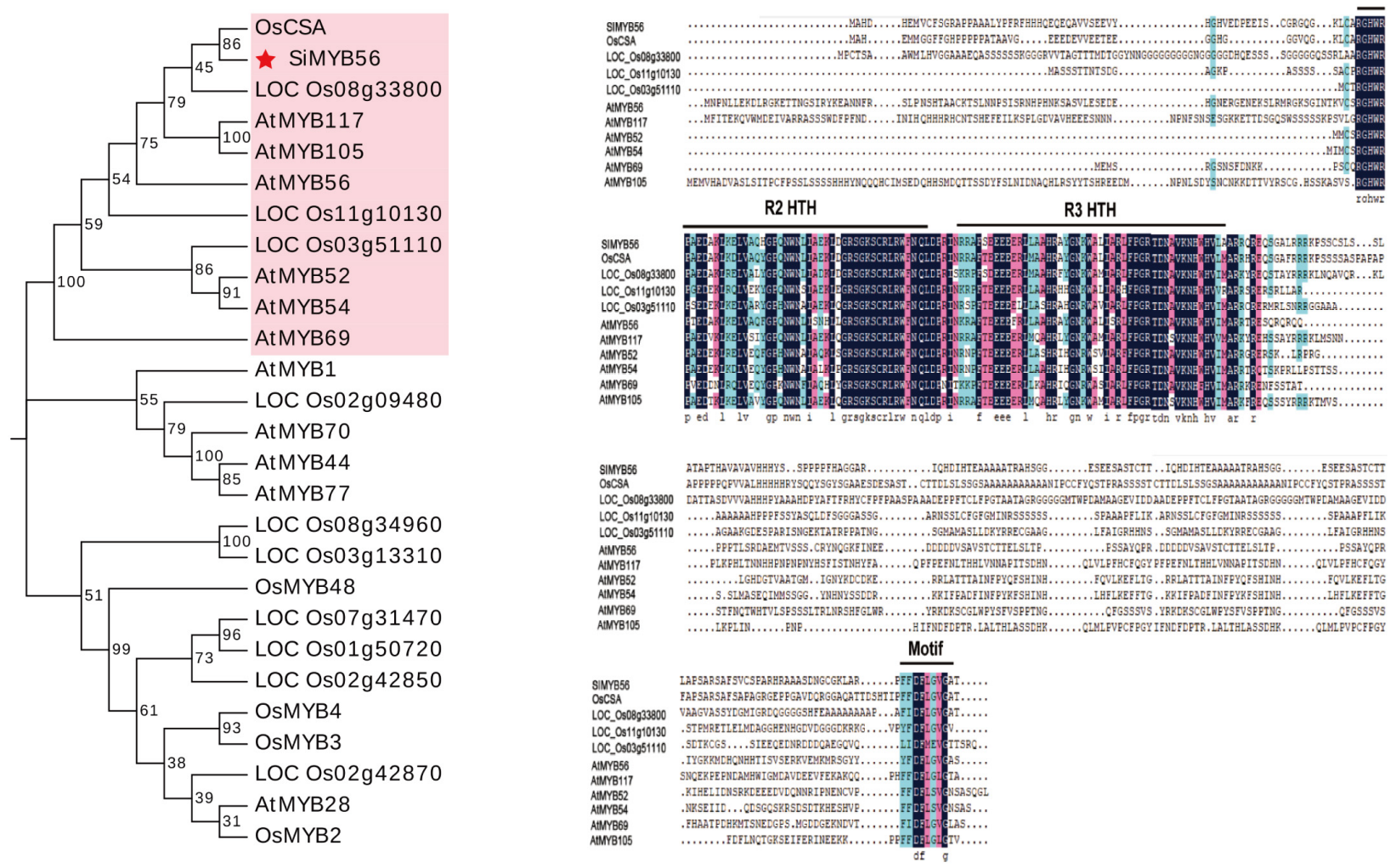

FIGURE 1 | Phylogenic and molecular analyses of SiMYB56. The filled red star indicates SiMYB56, and genes which are clustered in the same phylogenetic tree branch as SiMYB56 are labeled in pink. Two domains putatively responsible for the R2R3-MYB structure and a motif that belongs to Arabidopsis R2R2-MYB subgroup 21 are indicated. Identical amino acids are highlighted in blue.

two replicates (2017) or three replicates (2018). At 30 days after sowing, seedlings were randomly transplanted with 15$\times 30-\mathrm{cm}$ spacing and a single seeding per hill. Each line was in four $1.2 \mathrm{~m}$ long rows. One week after transplanted seedlings were established, irrigation was withheld for the rest of the growing season. When the rice matured, agronomic traits were determined.

\section{RNA Isolation and Quantitative Real-Time PCR}

Total RNA was extracted from seedlings using the Total RNA Extraction Kit (TIANGEN, China). The cDNA was synthesized in accordance with Fast Quant RT Super Mix Reverse Transcription Kit instructions (TransGene, Beijing, China). Real-time PCR amplification was performed using a Real Master Mix (SYBR Green, Beijing, China) kit (TransGene) and a fluorescence quantitative PCR instrument (ABI7500, United States). The relative gene expression in different samples was calculated using the $2^{-\Delta \Delta \mathrm{Ct}}$ method, using the $\mathrm{Ct}$ value at the specific fluorescence threshold for each sample. The actin genes for foxtail millet (Si001873m.g) and rice (LOC_Os03g50885) were used as internal controls. Quantitative real-time PCR was performed in triplicate. Primers used for quantitative real-time PCR are listed in Supplementary Table S1.

\section{Subcellular Localization Assay}

The full-length cDNA of SiMYB56 without stop codon were amplified and cloned into the BamHI site of vector 16318hGFP. The resulting SiMYB56-GFP fusion construct, driven by a CaMV35S promotor, and the positive control 16318hGFP were separately transformed into Arabidopsis mesophyll protoplasts using a PEG-calcium mediated method. This was followed by a $12-24 \mathrm{~h}$ incubation to allow transient expression. Chlorophyll autofluorescence and $\mathrm{H} 2 \mathrm{~B}$-mCherry were used as chloroplast and nuclear markers, respectively. The fluorescence in protoplast cells was visualized using a confocal microscope (Zeiss LSM700, Carl Zeiss, Oberkochen, Germany) and images were acquired with ZEN 2010 software (Carl Zeiss, Oberkochen, Germany). Arabidopsis protoplast cells were prepared based on a previous report (Yoo et al., 2007). Primers used are listed in Supplementary Table S1.

\section{Transcription Activation Assay in Yeast}

The SiMYB56 CDS sequence was amplified by PCR and fused in-frame with the GAL4 DNA binding domain by cloning into the NdeI site of vector pGBKT7 (Clontech). The constructs pGBKT7-SiMYB56 and the negative control pGBKT7 were separately transformed into yeast strain AH109 Saccharomyces cerevisiae through LiAc-mediated transformation 


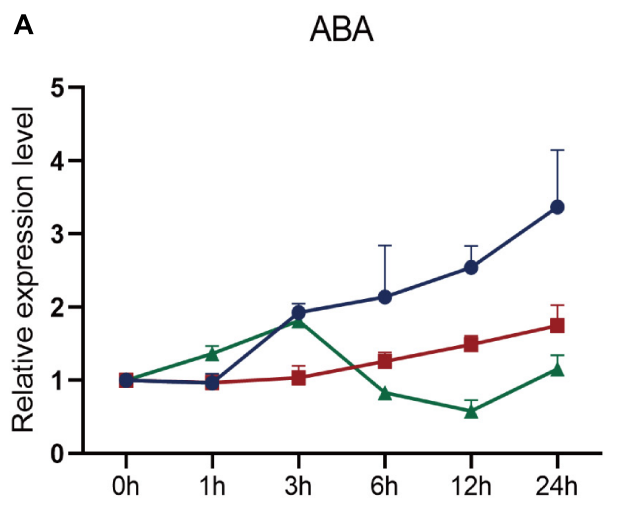

C

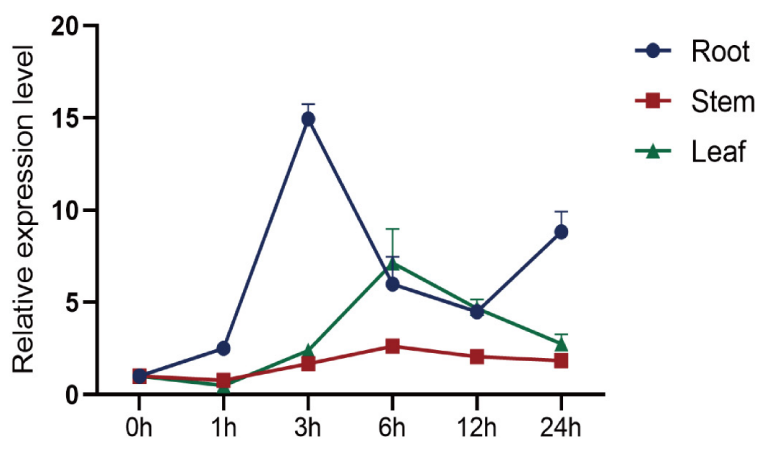

B

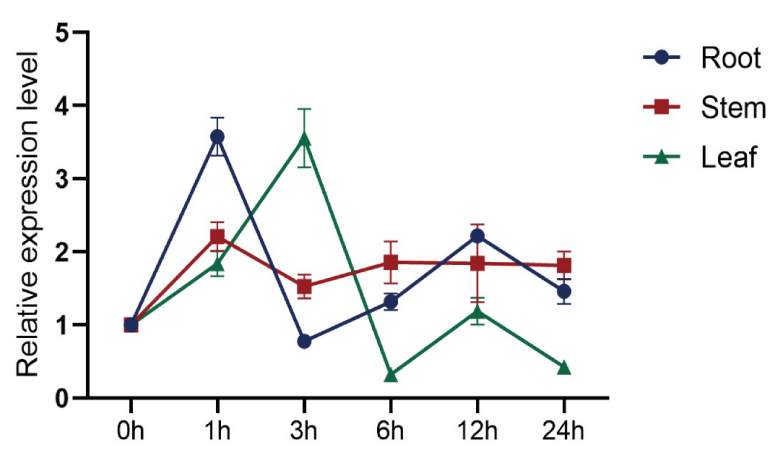

D

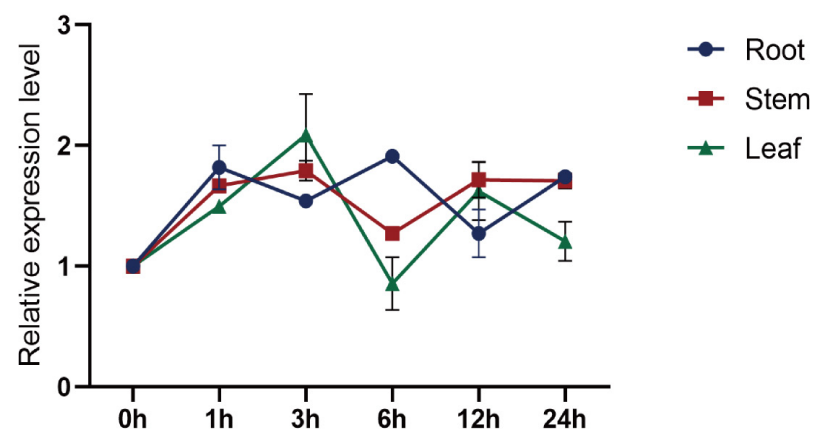

FIGURE 2 | Expression profile of SiMYB56. Relative SiMYB56 expression in foxtail millet under different treatments: (A) $100 \mu$ M ABA, (B) $10 \%$ PEG6000, (C) 80 mM $\mathrm{NaCl}$, and (D) Normal condition. All SiMYB56 expression levels were determined by qRT-PCR. Values shown are means \pm SD $(n=3)$.

according to the manufacturer's instructions (Clontech). Transformants was cultured on SD/-Trp medium at $28^{\circ} \mathrm{C}$. After 2 days, the positive transformants were plated on $\mathrm{SD} /-\mathrm{Trp}$ and SD/-Trp/-His/-Ade medium, respectively. The transcriptional activation activities were evaluated according to their growth status. Primers used are listed in Supplementary Table S1.

\section{Transcriptional Repression Assay in Protoplasts}

Transcriptional repression analysis was performed in protoplasts as described previously (Liu P. et al., 2018), For effectors vector, the coding sequences of SiMYB56 were first cloned into pGBKT7. Then the coding regions of BD-SiMYB56 fusion and $\mathrm{BD}$ were separately amplified and cloned into the PstI site of vector pGreenII 62-SK to get the effector plasmids (35S:BD-SiMYB56, 35S:BD). For the reporter vectors, the vector containing the firefly luciferase (LUC) reporter gene driven by the minimal TATA box plus five GAL4 binding elements and CaMV $35 \mathrm{~S}$ promoter was used for transcriptional repression test. The Renilla luciferase gene driven by the CaMV 35S promoter, was used as an internal control. The internal control, effector and reporter were simultaneously transformed into the protoplast cells, then kept in dark for $16 \mathrm{~h}$. The activities of LUC and REN were separately determined using Dual-Luciferase Reporter Assay System (Promega, E1910). Primers used are listed in Supplementary Table S1.

\section{Sequence Alignment, Phylogenetic, and Promoter Cis-Acting Element Analyses}

To investigate the relationship between SiMYB56 and other MYB transcription factors from Arabidopsis and rice, amino acid sequences were compared using DNAMAN software, and a systematic phylogenetic analysis was carried out using the neighbor-joining method in MEGA7.0 software with 1000 bootstrap replications. Amino acid sequences for Arabidopsis MYB TFs were acquired from TAIR $^{1}$ and those for rice MYB TFs were acquired from the Phytozome database ${ }^{2}$. For cis-element analysis, 2000 bp upstream of the translational start codon were extracted from Phytozome and examined as the promoter region. Potential promoter cis-elements for each gene were identified using the PlantCARE database ${ }^{3}$.

\footnotetext{
${ }^{1}$ https://www.arabidopsis.org/

${ }^{2}$ https://phytozome.jgi.doe.gov/pz/portal.html

${ }^{3}$ http://bioinformatics.psb.ugent.be/webtools/plantcare/html/
} 
A
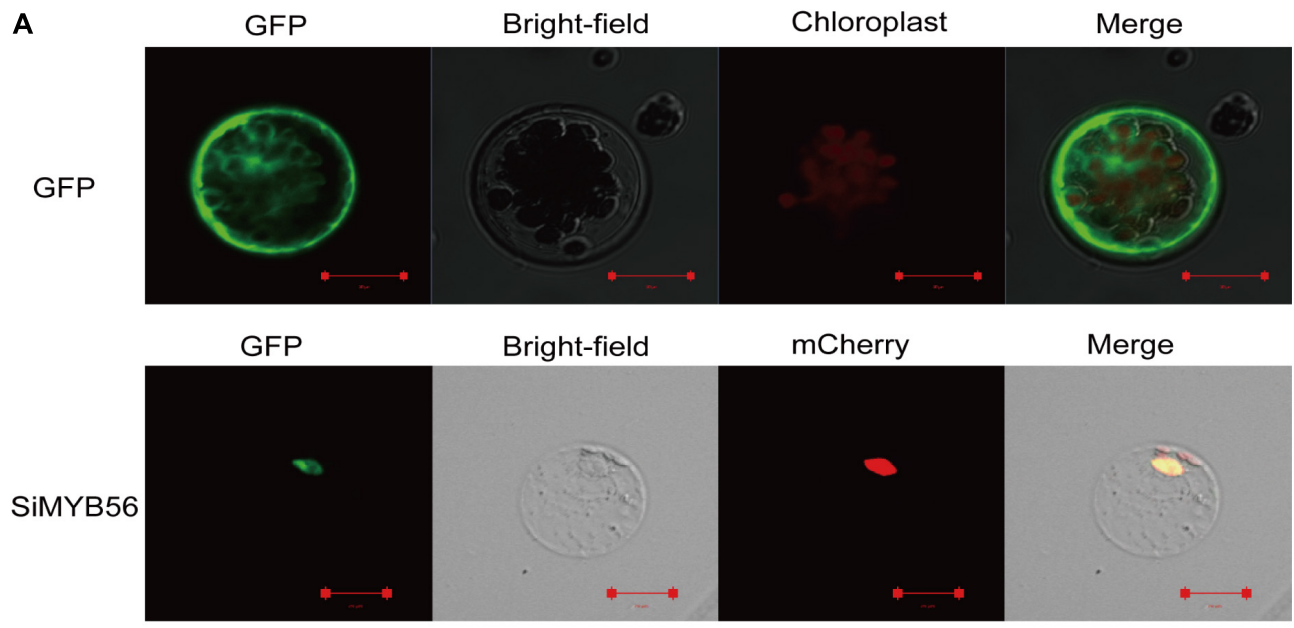

B

\section{Effectors}

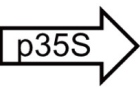

\section{GAL-BD}

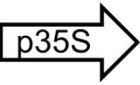

\section{GAL-BD}

\section{Reporters}

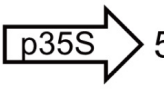

$5 \times$ GAL4-TATA- LUC

\begin{abstract}
Internal control
\end{abstract}

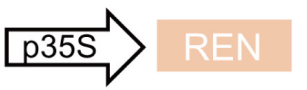

\section{SiMYB56}

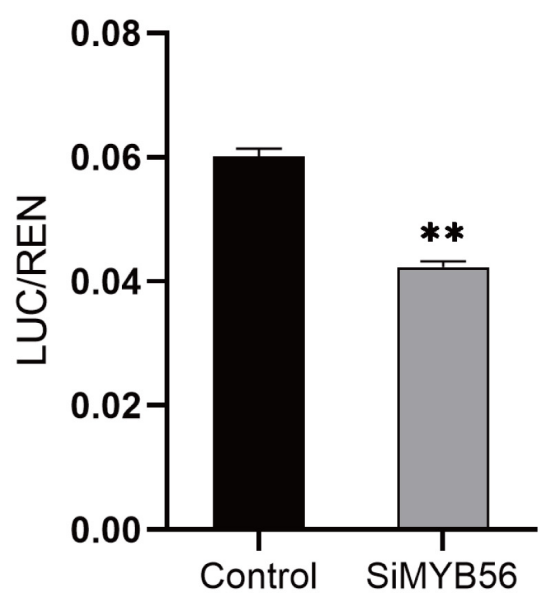

FIGURE 3 | Subcellular localization and transcription repression activity of SiMYB56. (A) Subcellular localization of SiMYB56 protein in Arabidopsis protoplasts. The 35S:SiMYB56-GFP and 35S:GFP control vectors were transiently expressed in Arabidopsis protoplasts, separately. Nuclear marker protein (H2B-mCherry) was co-expressed with 35S:SiMYB56-GFP. Fluorescence was observed using a Zeiss LSM 710 confocal microscope 16 h after transformation. (B) Transcription repression activity of SiMYB56 protein in Arabidopsis protoplasts. The activities of firefly luciferase (LUC) and renilla luciferase (REN) were determined $16 \mathrm{~h}$ post-transformation. The relative luciferase activities in control and SiMYB56-expressed samples were calculated by normalizing the LUC values against REN. Data represent the mean $\pm \mathrm{SD}(n=4),{ }^{\star \star} P \leq 0.01, t$-test.

\section{Transcriptional Activity Assays in Nicotiana benthamiana}

Transcriptional activity assays were performed in Nicotiana benthamiana leaves with reference to the methods described previously (Liu et al., 2017). The 2-kb promoters for 4CL5 and $\mathrm{F} 5 \mathrm{H} 1$ were fused with the luciferase reporter gene LUC in vector pGreenII0800 to generate reporter constructs 4CL5 $5_{\text {pro }}$ : LUC and $\mathrm{F} 5 \mathrm{H} 1_{\text {pro }}$ : LUC, respectively. Full-length SiMYB56 was separately cloned into the plant binary vector pCAMBIA1302 to generate the effector construct 35S:SiMYB56-GFP. The reporter and effector constructs were separately introduced into Agrobacterium strain GV3101(pSoup-p19), to carry out the coinfiltration (1:1) in Nicotiana benthamiana leaves. the LUC activities were observed and analyzed $48 \mathrm{~h}$ after infiltration using the Night SHADE LB 985 system (Berthold, Germany). After luciferin $(100 \mu \mathrm{M})$ spraying, leaves were kept in the dark for $5 \mathrm{~min}$, and then fluorescence was observed. Primers used are listed in Supplementary Table S1.

\section{Plasmid Construction and Rice Transformation}

The SiMYB56 coding region was amplified by PCR using the primers pMWB014-SiMYB56-F and pMWB014-SiMYB56-F. The PCR product was then ligated into the binary vector pMWB014 (Digested by BamHI) to obtain the construct Ubi:SiMYB56-FLAG. This construct was transformed into Oryza sativa cv. Kitaake using Agrobacterium-mediated transformation (Hiei et al., 1994). Seventeen transgenic rice lines were generated 

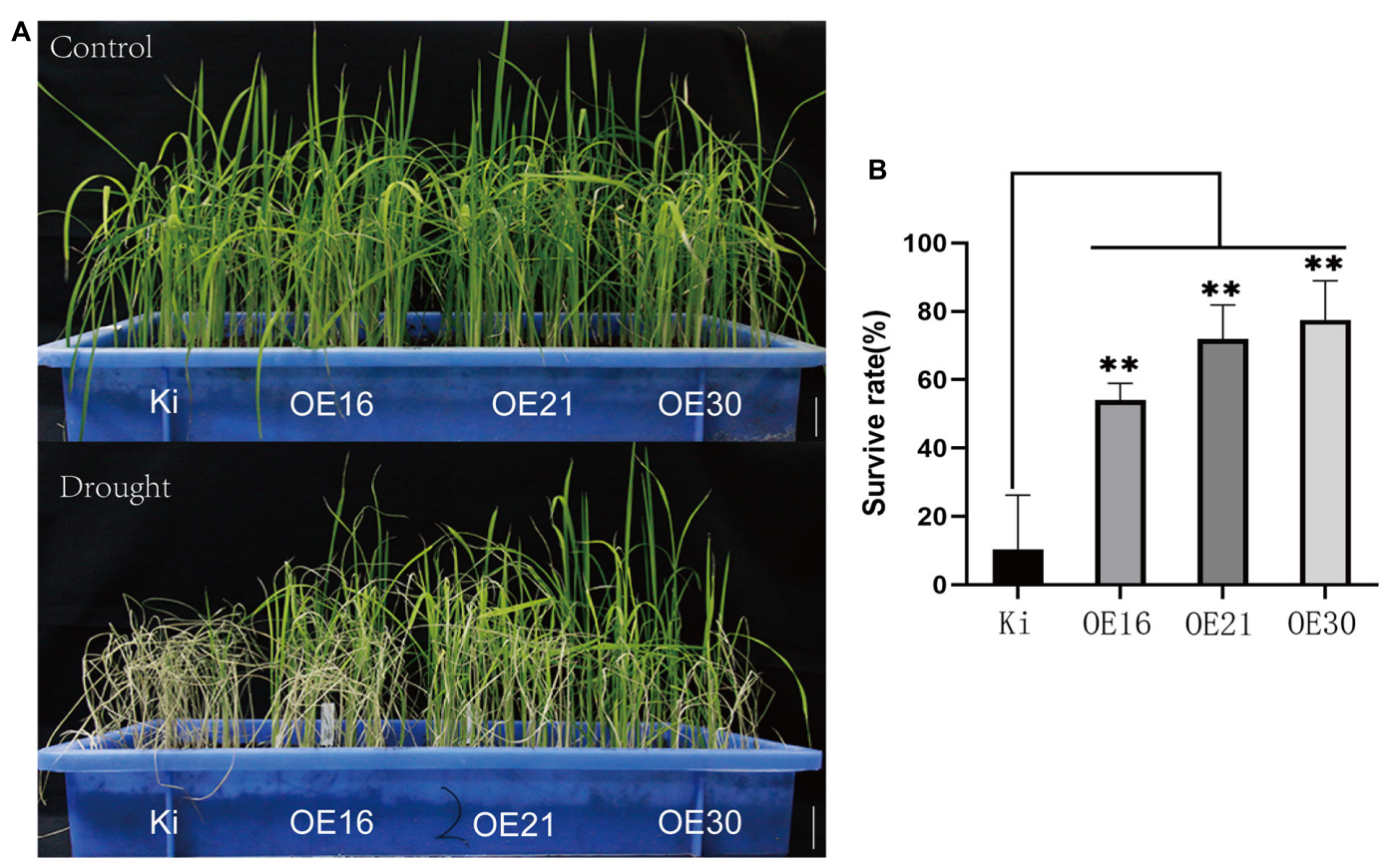

FIGURE 4 | Phenotypic analysis of transgenic rice plants and wild-type controls under drought conditions. (A) Growth of transgenic and wild-type rice seedlings under normal and drought conditions in a soil drought experiment. Bar $=5 \mathrm{~cm}$ (B) Survival rate of wild-type and transgenic rice plants after drought stress. Data represent the mean $\pm \operatorname{SD}(n=4),{ }^{\star \star} P \leq 0.01$, $t$-test.

and confirmed by PCR using primers Test-F (targeting the ubiquitin promoter) and Test-R (targeting the NOS terminator sequence). three transgenic lines (OE16, OE21, and OE30) were analyzed for phenotypes. Primers used are listed in Supplementary Table S1.

\section{Germination Assays}

For the germination assay, about 200 seeds from each of the transgenic and wild-type rice seeds were surface-sterilized in $10 \%(\mathrm{v} / \mathrm{v}) \mathrm{NaClO}$ for $15 \mathrm{~min}$, followed by washing five times with sterilized distilled water. Next, the transgenic and wildtype rice seeds (16 seeds, respectively) were evenly placed on the four corners of a plate covered with two layers of filter paper and then $0,2.5$, or $5 \mu \mathrm{M}$ ABA solution were add to the plate, respectively. The percentage of germinated seeds was calculated 2 days later. Each experiment included three replicates. One week later, germinated seed sprouts were sampled and frozen in liquid nitrogen for subsequent ABA content determination.

\section{Measurements of the ABA Content, MDA Content, Lignin Content, and Relative Electrolyte Leakage}

Measurements of ABA content: One-week-old sprouts (0.1 g) of transgenic and wild-type rice seeds germinated under the $0 \mu \mathrm{M}$ ABA treatment described above were extracted to measure ABA content. The experiments were performed in accordance with protocols for the Plant ABA ELISA Kit (Jianglaibio, JL13378-48T, Beijing, China). Each experiment included three replicates.
Measurements of MDA content: Leaves of 4-week-old seedlings $(0.1 \mathrm{~g})$ of transgenic and wild-type rice plants, grown under normal and 2-week 10\% PEG6000 treatments described above, were extracted to measure MDA content. The experiments were performed in accordance with MDA assay kit protocols (Comin, MDA-1-Y, China). Each experiment included three replicates.

Measurements of lignin content: Leaves of 4-week-old seedlings $(0.1 \mathrm{~g})$ of transgenic and wild-type rice plants grown under normal and 2-week 10\% PEG6000 treatments described above, were extracted to measure Lignin content. The experiments were performed following Lignin ELISA Kit protocols (Jianglaibio, JL22761-48T, Beijing, China). Each experiment included three replicates.

Measurements of relative electrolyte leakage: Leaves of 4week-old seedlings $(0.1 \mathrm{~g})$ of transgenic and wild-type rice plants grown under normal and 2-week 10\% PEG6000 treatments described above, were extracted to measure relative electrolyte leakage. The relative electrolyte leakage was examined in accordance with methods described in Cao et al. (2007).

\section{Histochemical Staining}

Hand-cut sections of 2-week-old leaves of transgenic rice plants and wild-type controls under normal condition or 3-week-old leaves of transgenic rice plants and wild-type controls under $10 \%$ PEG6000 treatment for about one week were soaked in an ethanol solution containing $1 \%(\mathrm{w} / \mathrm{v})$ phloroglucinol for $2 \mathrm{~min}$, and were then immersed in concentrated $\mathrm{HCl}$ for another $2 \mathrm{~min}$ (Herrera-Ubaldo and de Folter, 2018). The stained sections 

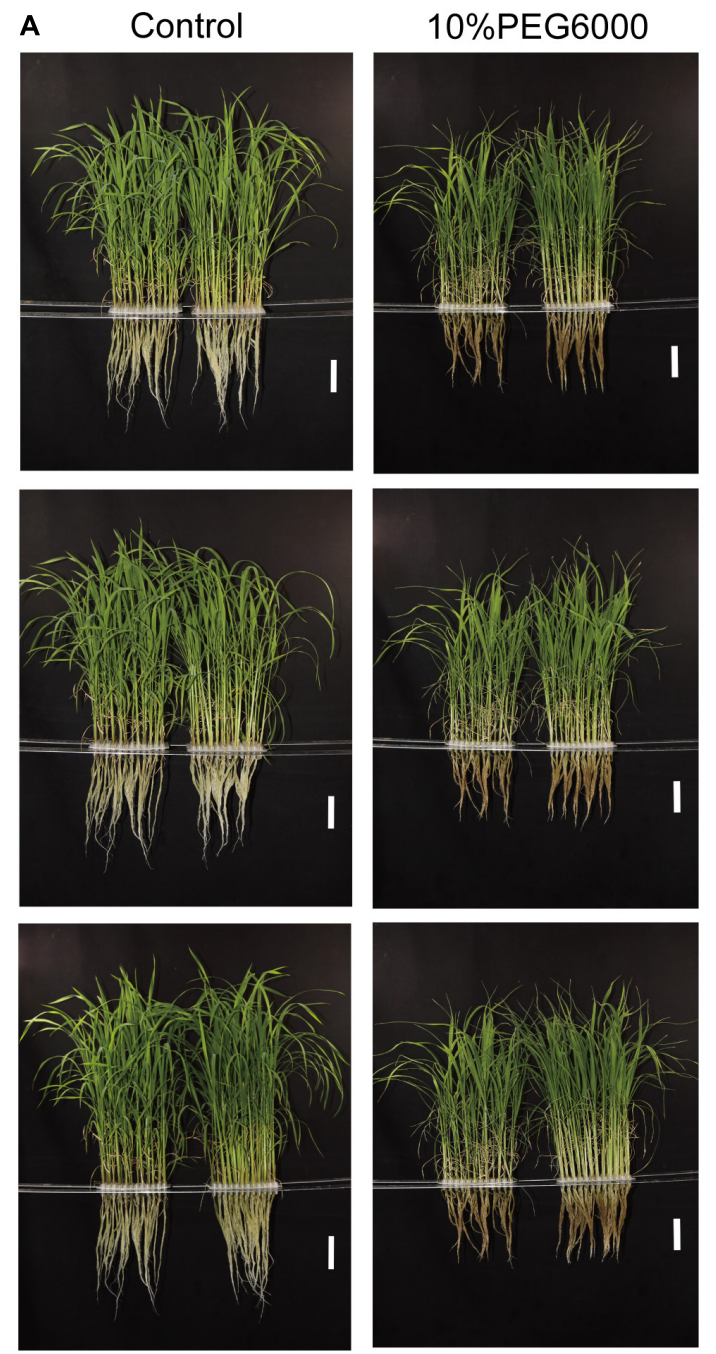

B
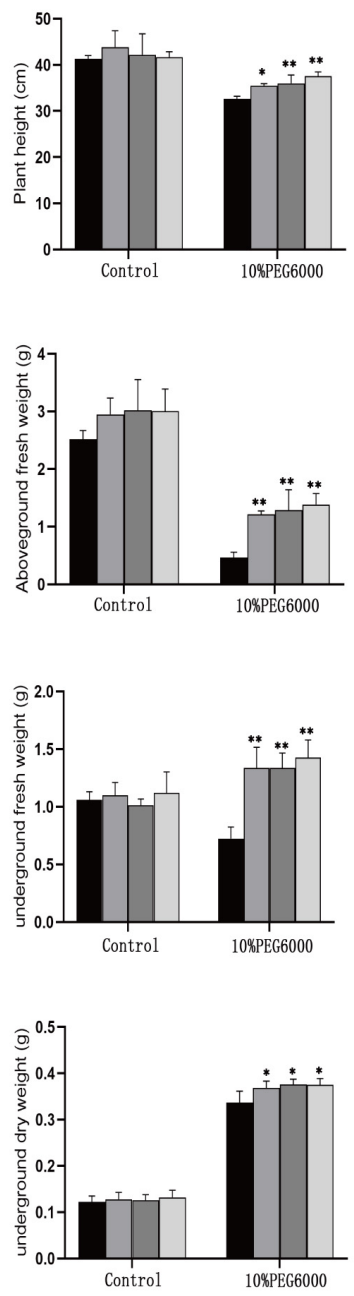

C
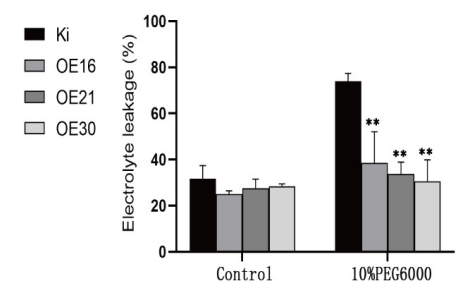

- $\mathrm{Ki}$

$\square$ OE16

$\square$ OE21

$\square$ OE30

D
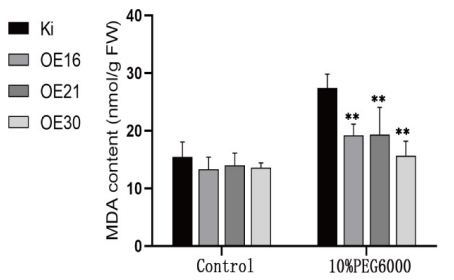

- $\mathrm{Ki}$

$\square$ OE16

$\square$ OE21

$\square$ OE30

E
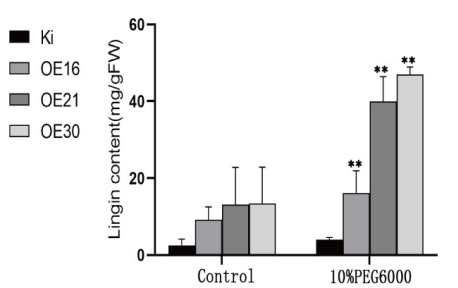

- $\mathrm{K}$

$\square$ OE16

$\square$ OE21

$\square$ OE30

$\square$ OE16

$\square$ OE21

$\square$ OE30

FIGURE 5 | Phenotypic analysis of transgenic rice plants and wild-type controls in response to 10\% PEG6000 treatments. (A) Phenotype of wild-type controls and transgenic rice plants under normal and 10\% PEG6000 conditions in a hydroponic drought experiment. Bar = $7 \mathrm{~cm}$. (B) Root length, plant height, aboveground fresh weight, underground fresh weight, aboveground dry weight, and underground dry weight under normal growth conditions and after $10 \%$ PEG6000 treatment. Relative electrolyte leakage (C), MDA content (D), and lignin content (E) under normal growth conditions and after $10 \%$ PEG6000 treatment. Data represent the mean $\pm \mathrm{SD}(n=4),{ }^{\star} P \leq 0.05,{ }^{\star \star} P \leq 0.01$, $t$-test.

were then examined using a Leica DMRB microscope (Leica, Wetzlar, Germany).

\section{RESULTS}

\section{SiMYB56 Belongs to Subgroup 21 of the R2R3-MYB Transcription Factor Family}

SiMYB56 is a 1296 bp long gene that encodes a putative 322 amino acid protein with a calculated molecular mass of $34.9 \mathrm{kD}$ and a $\mathrm{pI}$ of 8.31 (Supplementary Figure S1A) and is an R2R3-MYB transcription factor (Supplementary Figure S1B). Phylogenic and molecular analyses showed that SiMYB56 belongs to R2R3-MYB transcription factor subgroup 21 and is most closely related to rice CSA (Carbon Starved Anther) (Figure 1).

\section{Expression of SiMYB56 Can Be Induced by Various Abiotic Stresses}

To investigate the expression profile of SiMYB56, plantCARE was used to analyze its promoter region for putative regulatory elements. The analysis identified several cis-acting promoter elements that are related to drought resistance, salt tolerance, and ABA response, such as ABRE (Kaplan et al., 2006), DRE (Dubouzet et al., 2003), G-box, and W-box (Shen and Ho, 1995; Liu et al., 2016; Supplementary Figure S2). Quantitative realtime PCR showed that SiMYB56 expression was highest in the stem, lowest in the root (Supplementary Figure S3) and could be induced by various stresses (Figure 2A). Under ABA treatment, SiMYB56 transcript was induced in the root, increasing gradually with time (Figures 2A,D). Under PEG6000 stress, SiMYB56 transcript increased, peaking after a $1 \mathrm{~h}$ treatment in root and 


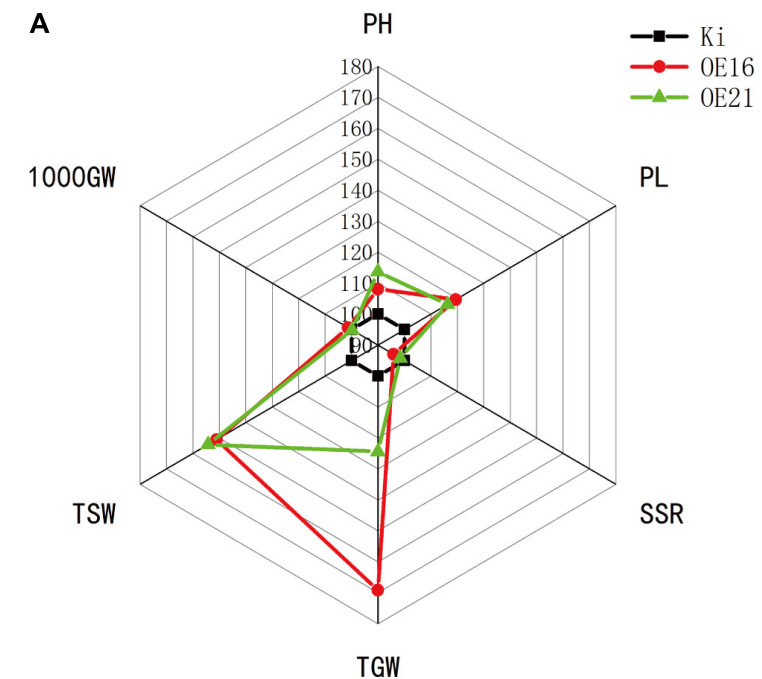

2017

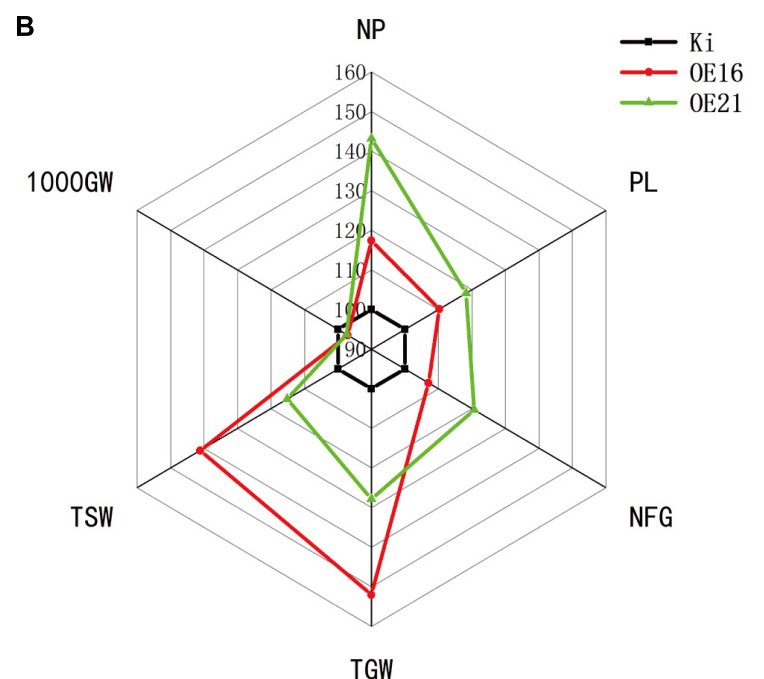

2018

FIGURE 6 | Phenotypic analysis of transgenic rice plants and wild-type controls grown under field-drought conditions. Spider plots of the agronomic traits of two independent homozygous T3 lines of transgenic rice plants and corresponding wild-type controls under field-drought conditions in year 2017 (A) and 2018 (B). Each data point represents the percentage of the mean values (2017, $n=2 ; 2018, n=3)$. The mean measurements from the wild-type controls were assigned a $100 \%$ reference value. $\mathrm{PH}$, plant height; SSR, seed setting rate; NP, number of panicles per hill; PL, panicle length; NFG, number of filled grains; TGW, total grain weight; TSW, total straw weight; 1000GW, 1,000 grain weight.

a $3 \mathrm{~h}$ treatment in leaf (Figures 2B,D). SiMYB56 transcript was strongly induced in root and leaf under $\mathrm{NaCl}$ treatment, increasing about 15-fold in root after a $3 \mathrm{~h}$ treatment and 5 -fold in leaf after a $6 \mathrm{~h}$ treatment (Figures 2C,D). The results of SiMYB56 expression profile analyses were consistent with promoter element predictions, indicating that SiMYB56 could indeed responds to various abiotic stresses in foxtail millet.

\section{SiMYB56 Protein Localizes to the Nucleus and Has Transcription Repression Activity}

To determine the subcellular localization of SiMYB56 protein, we generated SiMYB56-GFP fusion constructs controlled by the CaMV35S promoter. These constructs were then expressed in Arabidopsis mesophyll protoplasts, and plasmid 16318hGFP was used as a positive control. The results revealed that SiMYB56 fusion protein was expressed in the nucleus (Figure 3A), as confirmed by co-localization with a nuclear marker.

To test whether SiMYB56 protein had transcription activation or repression activity, the sequence of SiMYB56 was first fused in-frame to the GAL4 DNA-binding domain in the pGBKT7 vector, and the fusion construct pBD-SiMYB56 was transformed into yeast strain AH109. Plasmid pGBKT7 served as a negative control. Both pBD-SiMYB56 and pGBKT7 transformants grew on SD/-Trp but did not grow on SD/Trp/-Ade/-His (Supplementary Figure S4), indicating that SiMYB56 protein has no transcription activation activity. Then transcriptional repression assay in protoplasts was carried out to verify whether SiMYB56 protein has transcriptional repression activity. In this assay, the firefly luciferase (LUC) gene was fused to a $5 \times$ GAL4 binding site to generate the reporter, and the renilla luciferase (REN) gene driven by $35 \mathrm{~S}$ promoter was used as the internal control. Meanwhile, the effector plasmid was constructed by fusing the SiMYB56 coding sequence to the GAL4 DNA binding domain (BD). Bioluminescence determination revealed that the expression of SiMYB56 led to significant obvious down-regulation of the relative luciferase activity, compared to the control (Figure 3B). These results indicate that SiMYB56 has transcription repression activity.

\section{SiMYB56 Overexpression Significantly Improves Drought Tolerance of Transgenic Rice During the Vegetative Stage}

To evaluate SiMYB56 functions, drought stress tolerance analysis was carried out using homozygous T3 seeds of three transgenic rice lines (OE16/OE21/OE30) and wild-type rice (KITAKKE, Ki). Germinated seeds of homozygous transgenic rice were planted alongside germinated wild-type seeds. Two weeks later, irrigation was stopped for 15 days to subject rice plants to drought treatment. Then, the survival rate was calculated after allowing recovery following re-watering. The results showed no obvious differences in growth performance between transgenic rice plants and wild-type controls under normal growth conditions however, under drought treatments, the survival rate was significantly higher in three transgenic lines (50-80\%) than 
A

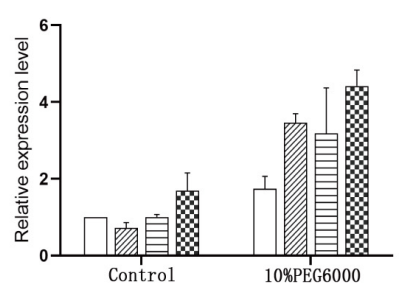

CCR10

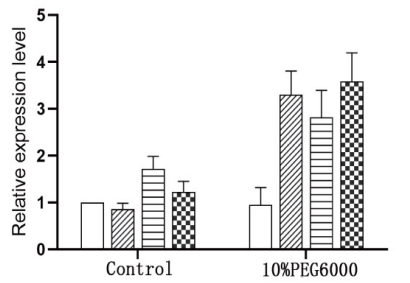

$\mathrm{C} 4 \mathrm{H}$

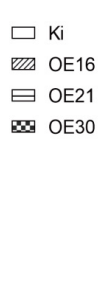

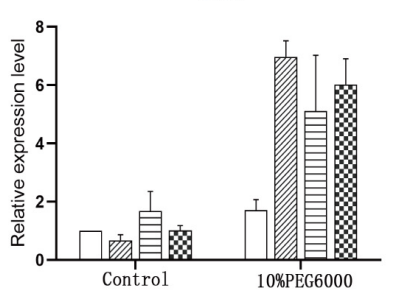

CAD
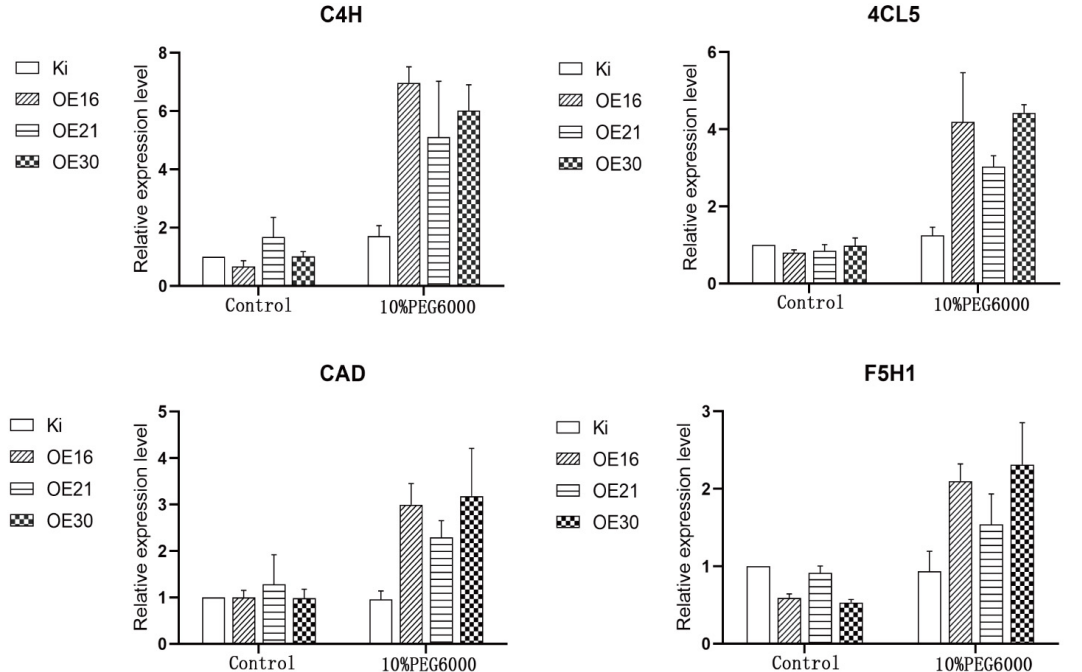

$$
\begin{aligned}
& \square \mathrm{Ki} \\
& \text { OE16 } \\
& \boxminus \mathrm{OE} 21 \\
& \mathrm{OE} 30
\end{aligned}
$$

F5H1
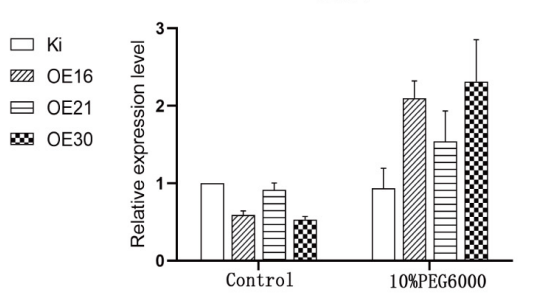

\section{B}
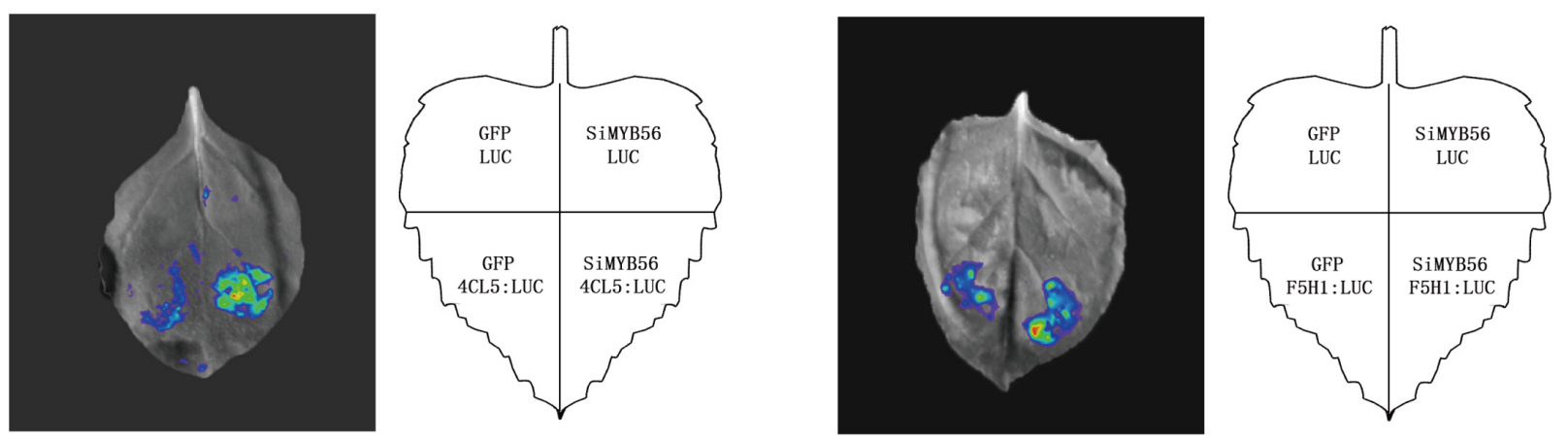

Low

High

Low

High

FIGURE 7 | SiMYB56 activates the expression of lignin synthesis related genes under drought conditions. (A) Expression analysis of lignin biosynthesis related genes in wild-type controls and transgenic rice plants. Entire 2-week-old SiMYB56-overexpressing and wild-type seedlings, growing under normal condition and 10\% PEG6000 treatment, were harvested for RNA isolation. Transcript level was quantified by qRT-PCR. Values shown are means \pm SD $(n=3)$. (B) Transcriptional activity assays in Nicotiana benthamiana leaves. SiMYB56 can activate the transcription of 4CL5 and F5H1. Four-week-old Nicotiana benthamiana plants were prepared for Agrobacterium-mediated transient expression, and generally the third through fifth leaf of each plant were analyzed. In each analysis, five independent Nicotiana benthamiana leaves were infiltrated and analyzed, and totally three biological replications were performed with quantification.

in wild-type rice (10\%) (Figures $4 \mathbf{A}, \mathbf{B}$ ), which suggested that SiMYB56 conferred drought stress tolerance in rice during the vegetative stage.

To further explore SiMYB56 functions in rice drought resistance, we performed another stress tolerance assay using 10\% PEG6000 to simulate drought stress (Figure 5A). Results showed that under 10\% PEG6000 treatment, root length, plant height, aboveground fresh weight, underground fresh weight, aboveground dry weight, and underground dry weight of transgenic rice plants were significantly higher than those of wild-type controls (Figure 5B). Results of electrolyte leakage and MDA content analyses showed that, under 10\% PEG6000 treatment conditions, transgenic rice plants had significantly lower electrolyte leakage and MDA content than wild-type controls, which indicated a lower degree of membrane damage in transgenic lines (Figures 5C,D). Phylogenic analyses have shown that SiMYB56 belongs to R2R3-MYB transcription factor subgroup 21. Many members of transcription factor subgroup 21 have been reported to be involved in the synthesis of secondary cell walls (Zhong et al., 2008; Zhang et al., 2012). Lignin, one of the main components of plant secondary cell walls, is closely related to plant drought resistance (Liu Q. et al., 2018). Therefore, lignin content of transgenic rice plants and wild-type controls was then analyzed, and the results showed that transgenic rice plants had significantly higher lignin content than wild-type controls under 10\% PEG6000 treatment conditions (Figure 5E and Supplementary Figure S5). These results demonstrate that SiMYB56 may improve transgenic rice drought resistance during the vegetative stage by increasing lignin biosynthesis and reducing membrane damage. 
A

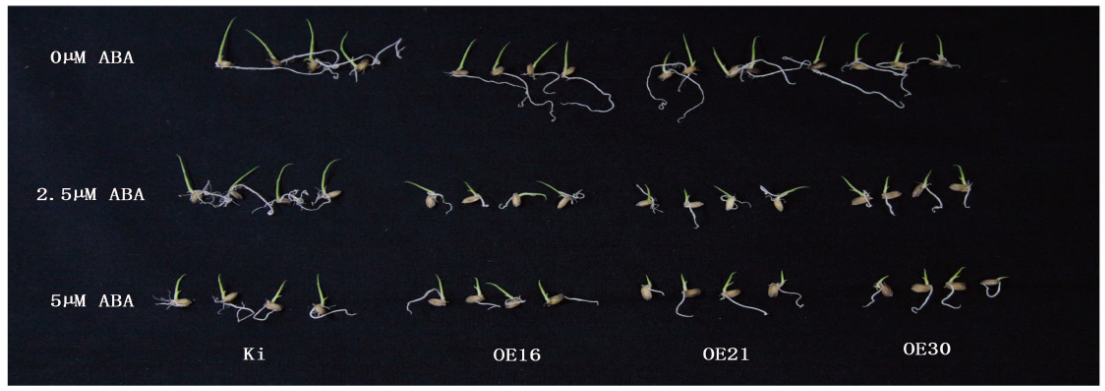

B

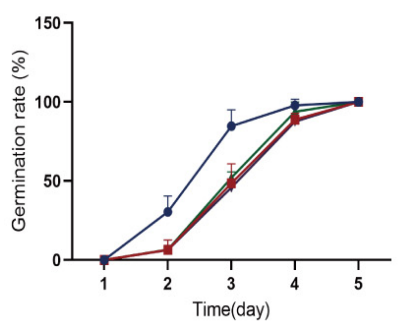

F

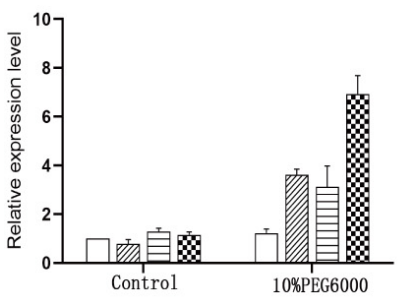

ABF2

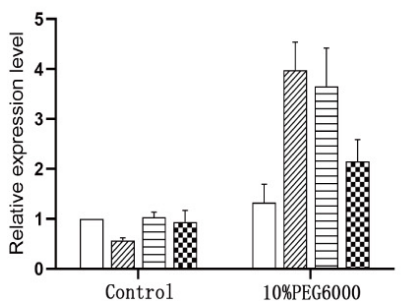

C

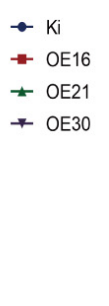

$\rightarrow \mathrm{Ki}$

OE16

OE30

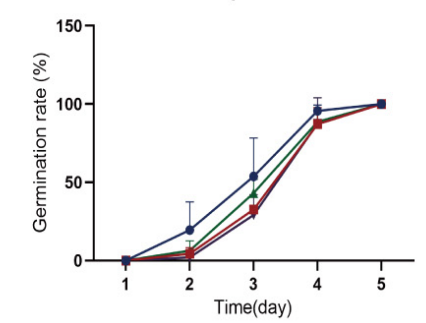

OSPK1

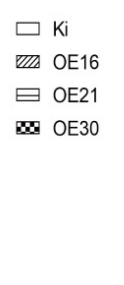

$\square \mathrm{Ki}$

四 OE16

$\boxminus$ OE21

B. OE30

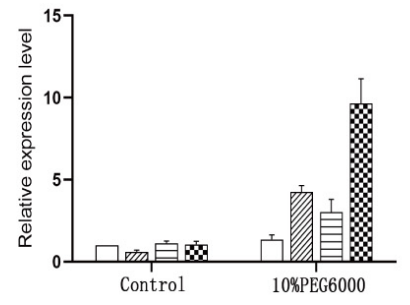

P5CS1

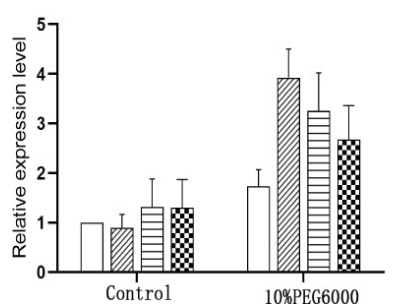

E

$0 \mu \mathrm{M}$ ABA

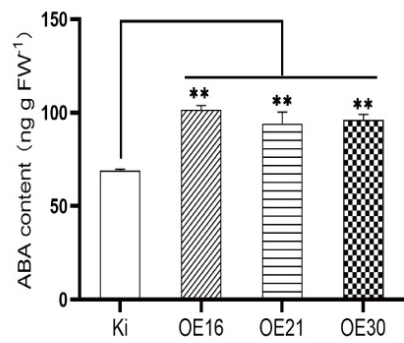

$\square \mathrm{Ki}$

WD OE16

$\boxminus$ OE21

W OE30 $\rightarrow \mathrm{Ki}$

$-\mathrm{OE} 16$

- OE21

+ OE30

D

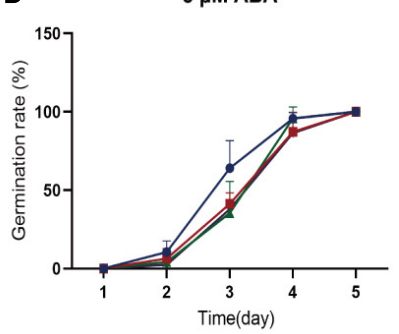

ABF1
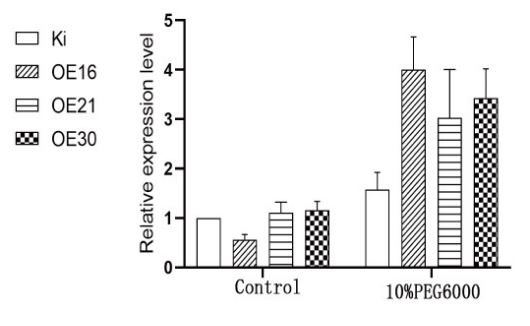

$\square \mathrm{Ki}$

UE16

$\boxminus \mathrm{OE} 21$

* OE30

FIGURE 8 | Overexpression of SiMYB56 significantly improves ABA accumulation in transgenic rice seeds and activates the expression of ABA signaling pathway related genes under drought condition. (A) Seeds of wild-type controls and transgenic rice plants were germinated on plates containing different ABA

concentrations. Germination rates of SiMYB56-overexpressing and wide-type seeds under $0 \mu \mathrm{M}$ (B), $2.5 \mu \mathrm{M}$ (C), and $5 \mu \mathrm{M}$ (D) ABA treatments $(n=3,16$ seeds in each replicate). (E) ABA content of sprouts from germinated seeds of transgenic rice plants and wide-type controls under $0 \mu \mathrm{M}$ ABA treatments. Values shown are means $\pm \mathrm{SD}(n=3)$. (F) Expression analysis of ABA signaling pathway related genes in wild-type controls and transgenic rice plants. Entire 2-week-old

SiMYB56-overexpressing and wild-type seedlings, grown under normal condition and 10\% PEG6000 treatments, were harvested for RNA isolation. Transcript level was quantified by qRT-PCR. Values shown are means \pm SD $(n=3)$.

\section{SiMYB56 Overexpression Increases Transgenic Rice Yield Under Field-Drought Conditions}

Crop yield under drought condition is closely related to the drought resistance of crops during the reproductive stage
(Boyer, 1982). Drought resistance experiments with seedlings proved that SiMYB56 conferred drought resistance to transgenic lines during the vegetative stage. In order to study SiMYB56 function during the reproductive stage, drought resistance experiments were carried out in the field in year 2017 and year 


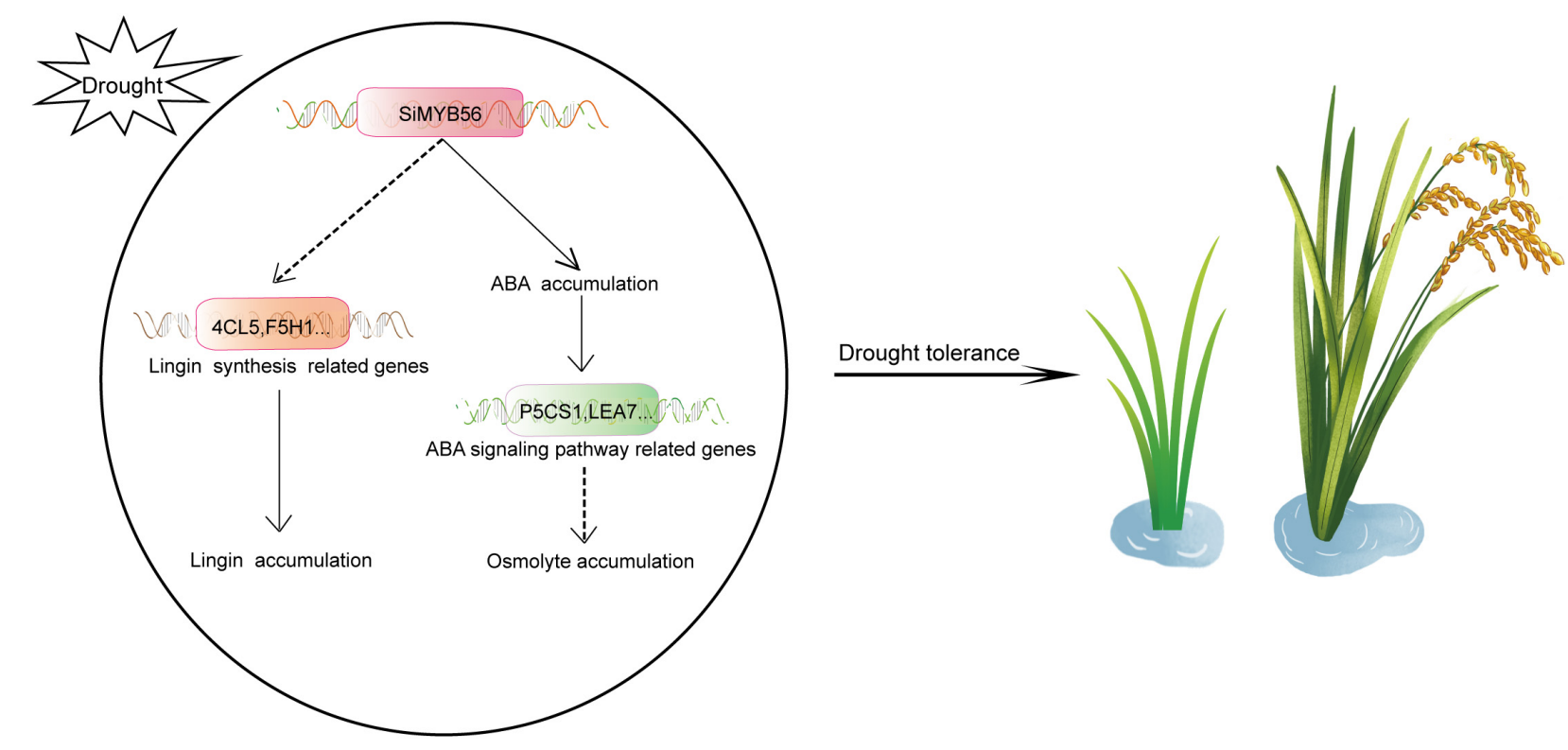

FIGURE 9| A proposed model for the role of SiMYB56 in drought tolerance regulation. Under drought stress, SiMYB56 expression is up-regulated, activating the expression of lignin synthesis related genes, such as $4 C L 5$ and $F 5 H 1$, which results in increased lignin content and subsequent drought tolerance. Up-regulation of SiMYB56 also increased ABA accumulation and upregulates ABA responsive genes, such as P5CS1 and LEA7, leading to osmolyte accumulation that supplements enhanced drought tolerance provided by increased lignin content.

2018. After harvesting, agronomic traits of transgenic rice plants and wild-type controls grown under drought conditions were measured which included plant height $(\mathrm{PH})$, seed setting rate (SSR), the number of panicles per plant (NP), panicle length (PL), number of filled grains (NFG), total grain weight (TGW), total straw weight (TSW), and 1000-grain weight (1000GW). Results showed that transgenic rice plants had higher total grain weight than wild-type rice under field-drought conditions both in year 2017 and 2018, which indicated that the drought resistance of SiMYB56 transgenic rice was higher than that of wild-type controls during the reproductive stage in the field. Moreover, we found that the increased yield for transgenic rice plants was mainly attributed to higher values of panicles per plant and panicle length which is also well reflected in the phenotype (Figures 6A,B and Supplementary Figure S6).

\section{SiMYB56 Overexpression Up-Regulated Lignin Biosynthesis Related Gene Expression Under Drought Conditions}

To elucidate the molecular mechanism of SiMYB56 transgenic rice enhanced drought tolerance, the expression of several lignin biosynthesis related genes, including $P A L, 4 C L, C 4 H, C C R, C A D$, and F5H (Minami et al., 1989; Yang et al., 2005; Kawasaki et al., 2006; Zhang et al., 2006; Gui et al., 2011; Takeda et al., 2017), were measured with quantitative real-time PCR. The results showed that there was no significant expression difference for six lignin biosynthesis related genes under normal conditions; whereas, under drought conditions, the expression of the six genes was 2-8 times higher in transgenic rice plants than in wild-type controls (Figure 7A). Next, to explore the regulatory effect of co-expressed SiMYB56 on transcription of PAL, 4CL5, $C 4 H, C C R 10, C A D$, and F5H1, Transcriptional activity assays were performed in Nicotiana benthamiana leaves with the promoters of these genes fused with a LUC reporter gene $\left(\mathrm{PAL}_{\mathrm{pro}}\right.$ : LUC, 4CL5 $5_{\text {pro }}$ : LUC, C4H pro: LUC, CCR10 $_{\text {pro }}$ : LUC, CAD pro: LUC, F5H1 $1_{\text {pro }}$ : LUC). Compared with empty vector (LUC), results showed that co-expression of 35S: SiMYB56 markedly increased the LUC expression driven by two promoters: 4CL5 and F5H1 (Figure 7B). These results suggested that SiMYB56 overexpression can increase the lignin content of transgenic plants by activating key lignin biosynthesis enzymes.

\section{Overexpression of SiMYB56 Significantly Improves ABA Accumulation in Transgenic Rice Seeds and Activates the Expression of ABA Signaling Pathway Related Genes Under Drought Conditions}

Abscisic acid can inhibit seed germination (Finkelstein et al., 2008). In order to explain the reduced seed germination rate of transgenic rice plants compared with wild-type controls, we analyzed seed germination rates of transgenic rice and wildtype seeds at different ABA concentrations (Figure 8A). Results showed that under normal conditions the germination rate of transgenic rice seeds was indeed lower than that of wild-type seeds (Figure 8B), while with $2.5 \mu \mathrm{M}$ (Figure 8C) and $5 \mu \mathrm{M} \mathrm{ABA}$ (Figure 8D) the germination rate difference between transgenic rice and wild-type seeds narrowed. Then, we determined the endogenous $\mathrm{ABA}$ content in the germinated seeds, and results 
showed that germinated seeds of transgenic rice plants had a higher content than those of wild-type controls (Figure 8E), which suggests that SiMYB56 could promote ABA synthesis in transgenic rice seeds.

Abscisic acid signaling pathway is important for plant response to drought stress (Yoshida et al., 2014), and SiMYB56 overexpression led to ABA accumulation. Therefore, we speculated that SiMYB56 could affect ABA signaling as another means of improving plant drought resistance. In order to test this hypothesis, we analyzed the expression of ABA synthesis related genes (NCED5), ABA signal transduction related genes (ABIL2, OSPK1, ABF1, ABF2, bZIP23), and ABA response related genes $(P 5 C S 1, L E A 7)$ in transgenic rice plants and wild-type controls under both normal and drought conditions (Figure 8F). Results showed that SiMYB56 enhanced ABA synthesis under drought conditions and activated the ABA signaling pathway, contributing to the enhanced drought tolerance of transgenic rice.

\section{DISCUSSION}

In this study, we demonstrated that SiMYB56 may enhance transgenic rice drought resistance both in greenhouse and field-drought conditions by activating the expression of lignin biosynthesis related genes lead to lignin accumulation (Figure 9). These results indicate that SiMYB56 can advantageously be used to improve the drought resistance of field-grown gramineous crops. Gramineous crops are the main food source for the world's population. With the aggravation of greenhouse gas effects and frequent drought, it is important to ensure global food security by improving the drought resistance of gramineous crops. Foxtail millet has a very high drought resistance, and regulation of lignin biosynthesis is an important part of that resistance. Increased lignification is a common response to biotic and abiotic stress (Moura et al., 2010). Studies have shown that many types of transcription factor improve plant drought resistance by regulating lignin biosynthesis. In rice, overexpression of OsTF1L, a rice HD-Zip transcription factor, promoted lignin biosynthesis and stomatal closure, which thereby improved drought tolerance (Bang et al., 2019). OsERF71, an AP2/ERF transcription factor, can modulate downstream genes, including general stress inducible genes, cell wall-associated genes, and lignin biosynthesis genes, further contributing to improved drought resistance (Lee et al., 2016). In white birch (Betula platyphylla), BpNAC012 increased salt and osmotic stress tolerance by regulating abiotic stress-responsive downstream genes, including D-1-pyrroline-5carboxylate synthetase, superoxide dismutase, and peroxidase, as well as secondary wall-associated downstream genes ( $\mathrm{Hu}$ et al., 2019). These results show that regulation of plant drought resistance by mediating lignin biosynthesis is a highly conservative process in plants and should be a future target of plant drought resistance improvement via molecular marker assisted breeding.

In addition to lignin accumulation, drought stress also triggers a series of stress-response pathways in plants (Zhu, 2002).
Under drought stress conditions, numerous stress response and tolerance genes are induced, and $\mathrm{ABA}$, a key plant stresssignaling hormone, accumulates (Tuteja, 2007). ABA controls plant stress response at multiple layers of regulation. These include: (1) transcriptional response, including interactions of core transcription factors that are regulated by $\mathrm{ABA}$ and other plant hormones; and (2) regulation of ABA metabolism and transport, with posttranscriptional and posttranslational regulation that remains a hidden and poorly recognized aspect of stress signaling (Daszkowska-Golec, 2016). Through a variety of signal transduction, ABA eventually triggers a series of plant physiological changes, such as stomatal closure, proline synthesis, and ROS scavenging (Stewart and Voetberg, 1985; Jiang and Zhang, 2001; Eisenach et al., 2017). These physiological changes improve plant drought resistance. In our study, SiMYB56 was shown to promote ABA synthesis, and quantitative real-time PCR indicated that SiMYB56 can activate a series of ABA signaling pathway related genes under drought conditions, including a proline synthesis gene (P5CS1) and a late embryogenesis abundant protein gene (LEA7). This result indicate that SiMYB56 may lead to osmolyte accumulation by activating ABA signaling (Figure 9), which in cooperation with lignin deposition improves transgenic rice drought resistance. ABA can rapidly accumulate in plants under drought stress to confer drought resistance, while lignin deposition mainly functions to relieve long-term drought stress (TerzI et al., 2013; Geng et al., 2018; Zhang et al., 2018). Our study showed that SiMYB56 may coordinate these two processes to improve rice drought resistance throughout a plant's lifecycle.

High lignin content affects the degradation and utilization of straw (Chen et al., 2015), and excessive ABA content inhibits normal plant growth (Chen et al., 2020). However, in phenotypic experiments, it was found that there was no significant difference in lignin content between transgenic plant lines and wild-type plants grown under normal conditions, and SiMYB56 overexpression did not affect rice growth under normal conditions. Quantitative real-time PCR also showed that the expression of genes related to lignin and ABA synthesis was upregulated only under drought conditions, which means SiMYB56 overexpression can only activate lignin and ABA synthesis under specific conditions. The mechanism by which SiMYB56 functions under drought stress needs to be further explored, but it seems clear that SiMYB56 overexpression does not affect normal plant growth.

\section{ACCESSION NUMBERS}

Sequence data from this article can be found in the Phytozome database under the following accession numbers: Seita.5G043900 (SiMYB56), LOC_Os02g41650 (PAL), LOC_Os08g34790 (4CL5), LOC_Os02g26770 (C4H), LOC_Os02g56700 (CCR10), LOC_Os08g43550 (CAD), LOC_Os10g36848 (F5H1), LOC_Os12g42280 (NCED5), LOC_Os05g51510 (ABIL2), LOC_Os02g34600 (OSPK1), LOC_Os01g64730 (ABF1), LOC_Os06g10880 (ABF2), LOC_Os02g52780 (bZIP23), LOC_Os05g38150 (P5CS1), and LOC_Os03g62620 (LEA7). 


\section{DATA AVAILABILITY STATEMENT}

All datasets presented in this study are included in the article/Supplementary Material.

\section{AUTHOR CONTRIBUTIONS}

WX designed and performed the experiments and wrote the manuscript. WT helped with the experiments. CW contributed to the implementation of the study. LG helped to clone the SiMYB56 gene. JS performed the field experiments. XQ and $\mathrm{ZH}$ contributed the valuable discussion. JC, YZ, and ZX provided instruction for the experiments. MC coordinated the project, conceived and designed the experiments, and edited the manuscript. Y-ZM coordinated the project and edited the manuscript. All authors have read and approved the final manuscript.

\section{REFERENCES}

Abe, H., Urao, T., Ito, T., Seki, M., Shinozaki, K., and Yamaguchi-Shinozaki, K. (2003). Arabidopsis AtMYC2 (bHLH) and AtMYB2 (MYB) function as transcriptional activators in abscisic acid signaling. Plant Cell 15, 63-78. doi: 10.1105/tpc.006130

Baldoni, E., Genga, A., and Cominelli, E. (2015). Plant MYB transcription factors: their role in drought response mechanisms. Int. J. Mol. Sci. 16, 15811-15851. doi: 10.3390/ijms160715811

Bang, S. W., Lee, D. K., Jung, H., Chung, P. J., Kim, Y. S., Choi, Y. D., et al. (2019). Overexpression of OsTF1L, a rice HD-Zip transcription factor, promotes lignin biosynthesis and stomatal closure that improves drought tolerance. Plant Biotechnol. J. 17, 118-131. doi: 10.1111/pbi.12951

Barton, L., Newsome, S. D., Chen, F. H., Wang, H., Guilderson, T. P., and Bettinger R. L. (2009). Agricultural origins and the isotopic identity of domestication in northern China. Proc. Natl. Acad. Sci. U.S.A. 106, 5523-5528. doi: 10.1073/pnas. 0809960106

Boyer, J. S. (1982). Plant productivity and environment. Science 218, 443-448 doi: $10.1126 /$ science.218.4571.443

Cao, W. H., Liu, J., He, X. J., Mu, R. L., Zhou, H. L., Chen, S. Y., et al. (2007) Modulation of ethylene responses affects plant salt-stress responses. Plant Physiol. 143, 707-719. doi: 10.1104/pp.106.094292

Chen, K., Li, G. J., Bressan, R. A., Song, C. P., Zhu, J. K., and Zhao, Y. (2020) Abscisic acid dynamics, signaling, and functions in plants. J. Integr. Plant Biol. 62, 25-54. doi: 10.1111/jipb.12899

Chen, Y., Huang, J., Li, Y., Zeng, G., Zhang, J., Huang, A., et al. (2015). Study of the rice straw biodegradation in mixed culture of Trichoderma viride and Aspergillus niger by GC-MS and FTIR. Environ. Sci. Pollut. Res. Int. 22, $9807-$ 9815. doi: 10.1007/s11356-015-4149-8

Daszkowska-Golec, A. (2016). "The role of abscisic acid in drought stress: how ABA helps plants to cope with drought stress," in Drought Stress Tolerance in Plants, Vol 2: Molecular and Genetic Perspectives, eds M. A. Hossain, S. H. Wani, S. Bhattacharjee, D. J. Burritt, and L.-S. P. Tran (Cham: Springer International Publishing), 123-151. doi: 10.1007/978-3-319-32423-4 5

Diao, X., Schnable, J., Bennetzen, J. L., and Li, J. (2014). Initiation of setaria as a model plant. Front. Agr. Sci. Eng. 1, 16-20. doi: 10.15302/j-fase- 2014011

Doust, A. N., Kellogg, E. A., Devos, K. M., and Bennetzen, J. L. (2009). Foxtail millet: a sequence-driven grass model system. Plant Physiol. 149, 137-141. doi: $10.1104 /$ pp.108.129627

Dubouzet, J. G., Sakuma, Y., Ito, Y., Kasuga, M., Dubouzet, E. G., Miura, S., et al. (2003). OsDREB genes in rice, Oryza sativa L., encode transcription activators that function in drought-, high-salt- and cold-responsive gene expression. Plant J 33, 751-763. doi: 10.1046/j.1365-313x.2003.01661.x

Eisenach, C., Baetz, U., Huck, N. V., Zhang, J., De Angeli, A., Beckers, G. J. M., et al. (2017). ABA-induced stomatal closure involves ALMT4, a

\section{FUNDING}

This work was supported by Key Projects of Genetic Modification (2018ZX08009-17B), the National Key Project for Research on Transgenic Biology (2016ZX08002-002), and the Agricultural Science and Technology Innovation Program (ASTIP, Transgenic Technology and Application of Crops, and Development and Application of Molecular Markers in Crops).

\section{SUPPLEMENTARY MATERIAL}

The Supplementary Material for this article can be found online at: https://www.frontiersin.org/articles/10.3389/fpls.2020.00785/ full\#supplementary-material

Phosphorylation-dependent vacuolar anion channel of Arabidopsis. Plant Cell 29, 2552-2569. doi: 10.1105/tpc.17.00452

Finkelstein, R. (2013). Abscisic acid synthesis and response. Arabidopsis Book 11:e0166. doi: 10.1199/tab.0166

Finkelstein, R., Reeves, W., Ariizumi, T., and Steber, C. (2008). Molecular aspects of seed dormancy. Annu. Rev. Plant Biol. 59, 387-415. doi: 10.1146/annurev. arplant.59.032607.092740

Ge, L., Dou, Y., Li, M., Qu, P., He, Z., Liu, Y., et al. (2019). SiMYB3 in foxtail millet (Setaria italica) confers tolerance to low-nitrogen stress by regulating root growth in transgenic plants. Int. J. Mol. Sci. 20:5741. doi: 10.3390/ijms20225741

Geng, D., Chen, P., Shen, X., Zhang, Y., Li, X., Jiang, L., et al. (2018). MdMYB88 and MdMYB124 enhance drought tolerance by modulating root vessels and cell walls in apple. Plant Physiol. 178, 1296-1309. doi: 10.1104/pp.18.00502

Gu, S. L., Liu, J., and Ren, H. R. (1987). Foxtail Millet Cultivation In China. Bejing. China Agricultural Press.

Gui, J., Shen, J., and Li, L. (2011). Functional characterization of evolutionarily divergent 4-coumarate:coenzyme a ligases in rice. Plant Physiol. 157, 574-586. doi: $10.1104 / \mathrm{pp} .111 .178301$

Herrera-Ubaldo, H., and de Folter, S. (2018). Exploring cell wall composition and modifications during the development of the gynoecium medial domain in Arabidopsis. Front. Plant Sci. 9:454. doi: 10.3389/fpls.2018.00454

Hiei, Y., Ohta, S., Komari, T., and Kumashiro, T. (1994). Efficient transformation of rice (Oryza sativa L.) mediated by Agrobacterium and sequence analysis of the boundaries of the T-DNA. Plant J. 6, 271-282. doi: 10.1046/j.1365-313x.1994. 6020271.x

$\mathrm{Hu}$, P., Zhang, K., and Yang, C. (2019). BpNAC012 positively regulates abiotic stress responses and secondary wall biosynthesis. Plant Physiol. 179, 700-717. doi: 10.1104/pp.18.01167

Hu, Y., Li, W. C., Xu, Y. Q., Li, G. J., Liao, Y., and Fu, F. L. (2009). Differential expression of candidate genes for lignin biosynthesis under drought stress in maize leaves. J. Appl. Genet. 50, 213-223. doi: 10.1007/BF03195675

Jiang, M., and Zhang, J. (2001). Effect of abscisic acid on active oxygen species, antioxidative defence system and oxidative damage in leaves of maize seedlings. Plant Cell Physiol. 42, 1265-1273. doi: 10.1093/pcp/pce162

Kaplan, B., Davydov, O., Knight, H., Galon, Y., Knight, M. R., Fluhr, R., et al. (2006). Rapid transcriptome changes induced by cytosolic Ca2+ transients reveal ABRE-related sequences as Ca2+-responsive cis elements in Arabidopsis. Plant Cell 18, 2733-2748. doi: 10.1105/tpc.106.042713

Kawasaki, T., Koita, H., Nakatsubo, T., Hasegawa, K., Wakabayashi, K., Takahashi, H., et al. (2006). Cinnamoyl-CoA reductase, a key enzyme in lignin biosynthesis, is an effector of small GTPase Rac in defense signaling in rice. Proc. Natl. Acad. Sci. U.S.A. 103, 230-235. doi: 10.1073/pnas.050987 5103

Ko, J. H., Jeon, H. W., Kim, W. C., Kim, J. Y., and Han, K. H. (2014). The MYB46/MYB83-mediated transcriptional regulatory programme is a 
gatekeeper of secondary wall biosynthesis. Ann. Bot. 114, 1099-1107. doi: 10 1093/aob/mcu126

Kubo, M., Udagawa, M., Nishikubo, N., Horiguchi, G., Yamaguchi, M., Ito, J., et al. (2005). Transcription switches for protoxylem and metaxylem vessel formation. Genes Dev. 19, 1855-1860. doi: 10.1101/gad.1331305

Lee, D. K., Jung, H., Jang, G., Jeong, J. S., Kim, Y. S., Ha, S. H., et al. (2016). Overexpression of the OsERF71 transcription factor alters rice root structure and drought resistance. Plant Physiol. 172, 575-588. doi: 10.1104/pp.16.00379

Lesk, C., Rowhani, P., and Ramankutty, N. (2016). Influence of extreme weather disasters on global crop production. Nature 529, 84-87. doi: 10.1038/ nature 16467

Li, W., Wang, K., Chern, M., Liu, Y., Zhu, Z., Liu, J., et al. (2020). Sclerenchyma cell thickening through enhanced lignification induced by OsMYB30 prevents fungal penetration of rice leaves. New Phytol. 226, 1850-1863. doi: 10.1111/nph. 16505

Liu, J., Cheng, X., Liu, P., Li, D., Chen, T., Gu, X., et al. (2017). MicroRNA319regulated TCPs interact with FBHs and PFT1 to activate $\mathrm{CO}$ transcription and control flowering time in Arabidopsis. PLoS Genet. 13:e1006833. doi: 10.1371/ journal.pgen.1006833

Liu, L., Xu, W., Hu, X., Liu, H., and Lin, Y. (2016). W-box and G-box elements play important roles in early senescence of rice flag leaf. Sci. Rep. 6:20881 doi: $10.1038 /$ srep20881

Liu, P., Liu, J., Dong, H., and Sun, J. (2018). Functional regulation of Q by microRNA172 and transcriptional co-repressor TOPLESS in controlling bread wheat spikelet density. Plant Biotechnol. J. 16, 495-506. doi: 10.1111/pbi.12790

Liu, Q., Luo, L., and Zheng, L. (2018). Lignins: biosynthesis and biological functions in plants. Int. J. Mol. Sci. 19:335. doi: 10.3390/ijms19020335

Lu, H., Zhang, J., Liu, K. B., Wu, N., Li, Y., Zhou, K., et al. (2009). Earliest domestication of common millet (Panicum miliaceum) in East Asia extended to 10,000 years ago. Proc. Natl. Acad. Sci. U.S.A. 106, 7367-7372. doi: 10.1073/ pnas.0900158106

McCarthy, R. L., Zhong, R., and Ye, Z. H. (2009). MYB83 is a direct target of SND1 and acts redundantly with MYB46 in the regulation of secondary cell wall biosynthesis in Arabidopsis. Plant Cell Physiol. 50, 1950-1964. doi: 10.1093/pcp/ pcp139

Minami, E., Ozeki, Y., Matsuoka, M., Koizuka, N., and Tanaka, Y. (1989). Structure and some characterization of the gene for phenylalanine ammonialyase from rice plants. Eur. J. Biochem. 185, 19-25. doi: 10.1111/j.14321033.1989.tb15075.x

Mitsuda, N., Iwase, A., Yamamoto, H., Yoshida, M., Seki, M., Shinozaki, K., et al. (2007). NAC transcription factors, NST1 and NST3, are key regulators of the formation of secondary walls in woody tissues of Arabidopsis. Plant Cell 19, 270-280. doi: 10.1105/tpc.106.047043

Moura, J. C., Bonine, C. A., de Oliveira, F. V. J., Dornelas, M. C., and Mazzafera, P. (2010). Abiotic and biotic stresses and changes in the lignin content and composition in plants. J. Integr. Plant Biol. 52, 360-376. doi: 10.1111/j.17447909.2010.00892.x

Muthamilarasan, M., Khandelwal, R., Yadav, C. B., Bonthala, V. S., Khan, Y., and Prasad, M. (2014). Identification and molecular characterization of MYB transcription factor superfamily in C4 model plant foxtail millet (Setaria italica L.). PLoS One 9:e109920. doi: 10.1371/journal.pone.0109920

Muthamilarasan, M., and Prasad, M. (2015). Advances in Setaria genomics for genetic improvement of cereals and bioenergy grasses. Theor. Appl. Genet. 128, 1-14. doi: 10.1007/s00122-014-2399-3

Ng, L. M., Melcher, K., Teh, B. T., and Xu, H. E. (2014). Abscisic acid perception and signaling: structural mechanisms and applications. Acta Pharmacol. Sin. 35, 567-584. doi: 10.1038/aps.2014.5

Ohtani, M., and Demura, T. (2019). The quest for transcriptional hubs of lignin biosynthesis: beyond the NAC-MYB-gene regulatory network model. Curr Opin. Biotechnol. 56, 82-87. doi: 10.1016/j.copbio.2018.10.002

Pereira, L., Domingues-Junior, A. P., Jansen, S., Choat, B., and Mazzafera, P. (2018). Is embolism resistance in plant xylem associated with quantity and characteristics of lignin? Trees Struct. Funct. 32, 349-358. doi: 10.1007/s00468017-1574-y

Sah, S. K., Reddy, K. R., and Li, J. (2016). Abscisic acid and abiotic stress tolerance in crop plants. Front. Plant Sci. 7:571. doi: 10.3389/fpls.2016.00571

Seo, P. J., Lee, S. B., Suh, M. C., Park, M. J., Go, Y. S., and Park, C. M. (2011). The MYB96 transcription factor regulates cuticular wax biosynthesis under drought conditions in Arabidopsis. Plant Cell 23, 1138-1152. doi: 10.1105/tpc. 111.083485

Shen, Q., and Ho, T. H. (1995). Functional dissection of an abscisic acid (ABA)inducible gene reveals two independent ABA-responsive complexes each containing a G-box and a novel cis-acting element. Plant Cell 7, 295-307. doi: $10.1105 /$ tpc.7.3.295

Shinozaki, K., and Yamaguchi-Shinozaki, K. (2007). Gene networks involved in drought stress response and tolerance. J. Exp. Bot. 58, 221-227. doi: 10.1093/ jxb/erl164

Stewart, C. R., and Voetberg, G. (1985). Relationship between stress-induced ABA and proline accumulations and ABA-induced proline accumulation in excised barley leaves. Plant Physiol. 79, 24-27. doi: 10.1104/pp.79.1.24

Takeda, Y., Koshiba, T., Tobimatsu, Y., Suzuki, S., Murakami, S., Yamamura, M., et al. (2017). Regulation of CONIFERALDEHYDE 5-HYDROXYLASE expression to modulate cell wall lignin structure in rice. Planta 246, 337-349. doi: $10.1007 / \mathrm{s} 00425-017-2692-x$

Terzi, R., Saruhan, N., Kutlu, ÇN., and ĞLu, A. (2013). Lignification response for rolled leaves of Ctenanthe setosa under long-term drought stress. Turk. J. Biol. 37, 614-619. doi: 10.3906/biy-1210-27

Tuteja, N. (2007). Abscisic Acid and abiotic stress signaling. Plant Signal. Behav. 2, 135-138. doi: 10.4161/psb.2.3.4156

Wang, Y., Sheng, L., Zhang, H., Du, X., An, C., Xia, X., et al. (2017). CmMYB19 over-expression improves aphid tolerance in chrysanthemum by promoting lignin synthesis. Int. J. Mol. Sci. 18:619. doi: 10.3390/ijms18030619

Xu, Z., Zhou, G., and Shimizu, H. (2010). Plant responses to drought and rewatering. Plant Signal. Behav. 5, 649-654. doi: 10.4161/psb.5.6.11398

Yang, D. H., Chung, B. Y., Kim, J. S., Kim, J. H., Yun, P. Y., Lee, Y. K., et al. (2005). cDNA cloning and sequence analysis of the rice Cinnamate-4-Hydroxylase gene, a cytochrome P450-dependent monooxygenase involved in the general phenylpropanoid pathway. J. Plant Biol. 48, 311-318. doi: 10.1007/bf03030528

Yoo, S. D., Cho, Y. H., and Sheen, J. (2007). Arabidopsis mesophyll protoplasts: a versatile cell system for transient gene expression analysis. Nat. Protoc. 2, 1565-1572. doi: 10.1038/nprot.2007.199

Yoshida, T., Mogami, J., and Yamaguchi-Shinozaki, K. (2014). ABA-dependent and ABA-independent signaling in response to osmotic stress in plants. Curr. Opin. Plant Biol. 21, 133-139. doi: 10.1016/j.pbi.2014.07.009

Zhang, F. P., Sussmilch, F., Nichols, D. S., Cardoso, A. A., Brodribb, T. J., and McAdam, S. A. M. (2018). Leaves, not roots or floral tissue, are the main site of rapid, external pressure-induced ABA biosynthesis in angiosperms. J. Exp. Bot. 69, 1261-1267. doi: 10.1093/jxb/erx480

Zhang, K., Qian, Q., Huang, Z., Wang, Y., Li, M., Hong, L., et al. (2006). GOLD HULL AND INTERNODE2 encodes a primarily multifunctional cinnamylalcohol dehydrogenase in rice. Plant Physiol. 140, 972-983. doi: 10.1104/pp.105. 073007

Zhang, L., Zhao, G., Jia, J., Liu, X., and Kong, X. (2012). Molecular characterization of 60 isolated wheat MYB genes and analysis of their expression during abiotic stress. J. Exp. Bot. 63, 203-214. doi: 10.1093/jxb/err264

Zhao, Q., and Dixon, R. A. (2011). Transcriptional networks for lignin biosynthesis: more complex than we thought? Trends Plant Sci. 16, 227-233. doi: 10.1016/j.tplants.2010.12.005

Zhong, R., Lee, C., Zhou, J., McCarthy, R. L., and Ye, Z. H. (2008). A battery of transcription factors involved in the regulation of secondary cell wall biosynthesis in Arabidopsis. Plant Cell 20, 2763-2782. doi: 10.1105/tpc.108. 061325

Zhu, J. K. (2002). Salt and drought stress signal transduction in plants. Annu. Rev. Plant Biol. 53, 247-273. doi: 10.1146/annurev.arplant.53.091401.143329

Conflict of Interest: The authors declare that the research was conducted in the absence of any commercial or financial relationships that could be construed as a potential conflict of interest.

Copyright (C) 2020 Xu, Tang, Wang, Ge, Sun, Qi, He, Zhou, Chen, Xu, Ma and Chen. This is an open-access article distributed under the terms of the Creative Commons Attribution License (CC BY). The use, distribution or reproduction in other forums is permitted, provided the original author(s) and the copyright owner(s) are credited and that the original publication in this journal is cited, in accordance with accepted academic practice. No use, distribution or reproduction is permitted which does not comply with these terms. 